\title{
Early tumor shrinkage as a predictive factor of metastatic renal cell carcinoma in molecular targeted therapy: A single institutional study
}

\author{
SUGURU SHIROTAKE ${ }^{1}$, HIDEYUKI KONDO ${ }^{1 *}$, TAKASHI OKABE ${ }^{1 *}$, SOICHI MAKINO $^{2 *}$, RYUICHIRO ARAKI $^{3}$, \\ AKARI KOMATSUDA $^{1}$, GO KANEKO ${ }^{1}$, KOSHIRO NISHIMOTO ${ }^{1}$ and MASAFUMI OYAMA ${ }^{1}$ \\ Departments of ${ }^{1}$ Uro-Oncology and ${ }^{2}$ Radiation Oncology, Saitama Medical University International Medical Center, Hidaka, \\ Saitama 350-1298; ${ }^{3}$ Saitama Medical University, Community Health Science Center, Moroyama, Saitama 350-0495, Japan
}

Received June 7, 2018; Accepted October 11, 2018

DOI: $10.3892 / \mathrm{mco} .2018 .1762$

\begin{abstract}
The aim of the present study was to investigate the impact of metastatic sites and early tumor shrinkage (eTS) as prognostic predictive factors of metastatic renal cell carcinoma (mRCC) in molecular targeted therapy. A total of 209 advanced RCC cases treated with sorafenib, sunitinib, axitinib, pazopanib, temsirolimus and everolimus from our single institution were included in the present study. Several known prognostic predictive factors, including metastatic sites and the rate of eTS, were analyzed by Kaplan-Meier survival estimate analysis followed by Cox's proportional hazards model analysis. eTS was measured by three independent physicians. Four metastatic sites in the liver, bone, lymph nodes and brain as well as greater eTS were identified as potential independent predictors of overall survival (OS) in several cohorts: i) Metastatic RCC ( $n=194)$; ii) metastatic clear cell RCC $(n=119)$; and iii) $\mathrm{mRCC}$ patients with eTS data $(n=127)$. In sub-analyses of patients treated with each 1st line tyrosine kinase inhibitor, eTS was identified as a potentially potent predictor of OS in patients treated with axitinib. The liver, bone, lymph nodes, brain metastases and eTS were identified as independent predictive factors of OS by analyzing a limited Japanese cohort.
\end{abstract}

\section{Introduction}

Renal cell carcinoma (RCC) encompasses a group of cancers that originate from renal tubular epithelial cells, and metastatic RCC (mRCC) is currently treated with molecular targeted

Correspondence to: Dr Koshiro Nishimoto or Dr Suguru Shirotake, Department of Uro-Oncology, Saitama Medical University International Medical Center, 1397-1 Yamane, Hidaka, Saitama 350-1298, Japan

E-mail: knishi@saitama-med.ac.jp

E-mail: ss197816@saitama-med.ac.jp

${ }^{*}$ Contributed equally

Key words: renal cell carcinoma, predictive factor, early tumor shrinkage, molecular targeted therapy therapy (MTT) agents (1). The MTT agent sorafenib was approved for the treatment of mRCC in Japan in 2008, and targets tyrosine kinases involved in angiogenesis, including platelet-derived growth factor and vascular endothelial growth factors (VEGF) $(2,3)$. Prior to its approval, in a period called the Cytokine Era (4), the systemic treatment of mRCC was limited to 1 st generation immunotherapy, i.e., treatments with interferon and interleukin 2. However, new agents were approved in Japan: Sunitinib (5), axitinib (6), pazopanib (7), everolimus (8), and temsirolimus (9). Sunitinib, axitinib, and pazopanib target similar molecules to sorafenib. Among these, axitinib has greater selectivity to VEGF receptors. In contrast, everolimus and temsirolimus inhibit the mammalian target of the rapamycin pathway. Axitinib, everolimus, and temsirolimus are generally used as 2nd line MTT. Nivolumab was approved as the first immune checkpoint inhibitor (2nd generation immunotherapy), and improved survival in patients with RCC (10).

Our institution, the Saitama Medical University International Medical Center, is one of the largest volume centers of high-risk mRCC patients in Japan. As of February 2017, we have treated 209 patients with MTT agents. Naito et al (4) previously reported predictive factors of $\mathrm{mRCC}$ in the Cytokine Era, and also indicated superior survival in the Japanese cohort compared to other countries. However, a similar study in the MTT era was not conducted in Japan. Furthermore, a novel concept, 'early tumor shrinkage (eTS)', was reported to predict survival under MTT (11-13). In the present study focused on eTS, we reviewed our MTT experience and identified predicting factors of $\mathrm{mRCC}$ in the MTT era before the introduction of 2nd generation immunotherapy era.

\section{Materials and methods}

Ethics and patient selection. The present study was approved by the Institutional Review Board of Saitama Medical University International Medical Center (approval no. 14-049). The clinical and pathological data of 209 patients with advanced RCC (with or without metastasis) treated with MTT between April 25, 2008 and February 27, 2017, were retrospectively isolated from electronic medical records using the terms 'sorafenib', 'sunitinib', 'axitinib', 'pazopanib', 'temsirolimus', 
'everolimus', 'renal tumor', and 'renal cell carcinoma'. Data on known risk factors including the pathology of nephrectomy and/or tumor biopsy, clinical findings including laboratory data and radiographic images, and whether patients underwent nephrectomy were manually isolated from electronic records. In order to predict the outcomes of mRCC patients treated with MTT, we stratified patients into three groups using six factors of 1st line therapy (i.e., time from diagnosis to treatment, Karnofsky performance status, hemoglobin level, neutrophil count, platelet count, and corrected calcium) based on the International Metastatic Renal Cell Carcinoma Database Consortium (IMDC) model (14) (Table I).

Routine examination and follow-up. In our institution, patients treated with MTT generally visited our office every 2-4 weeks. Radiographical evaluations using computed tomography (CT) and/or magnetic resonance imaging (MRI) were performed every 3-6 months, and additional CT and bone scintigraphy were conducted when clinically indicated. Patients without neurological symptoms were generally not subjected to brain imaging tests.

MTT administration and RDI. Patients generally began MTT at the recommended starting dose. Relative dose intensity (RDI) was assessed as the cumulative dose divided by the duration of therapy and optimal daily doses [sorafenib $800 \mathrm{mg} / \mathrm{day}$, sunitinib $33.3 \mathrm{mg} /$ day, axitinib $10 \mathrm{mg} /$ day, pazopanib $800 \mathrm{mg} / \mathrm{day}$, everolimus $10 \mathrm{mg} /$ day, and temsirolimus $3.57 \mathrm{mg} /$ day $(25 \mathrm{mg}$ infusion/week)].

eTS measurements. Among $194 \mathrm{mRCC}$ patients, the renal tumors or metastatic lesions of 127 were evaluated by CT or MRI 90 days before MTT treatment (as baseline scans) as well as between 30 and 90 days after MTT (as first post baseline scans). Two urologists (HK and TO) and a radiologist (SM) blindly measured the sum of the longest diameters (SLD) in each case, based on the Response Evaluation Criteria in Solid Tumors (RECIST) version 1.1 (15). Tumor shrinkage was determined based on the change in SLD of target lesions at the first post baseline scan. In order to estimate the power of eTS, cut-off values were used as follows; -0.2 (20\% decrease), -0.15 ( $15 \%$ decrease), -0.1 ( $10 \%$ decrease), -0.05 ( $5 \%$ decrease), 0.0 (no change), and +0.05 ( $5 \%$ only increase).

Outcomes. The purpose of the present study was to evaluate overall survival (OS), which was defined as the time from the initiation of 1st line targeted therapy to the date of death from any cause or the date of the last follow-up. Radiographic responses were assessed by radiographic criteria according to RECIST version 1.1. We retrospectively obtained outcome and survival data, while cause of death was identified by the attending physicians, chart reviews corroborated by death certificates, or death certificates alone at other institutions.

Statistical analysis. The variables of different groups were compared by the Friedman test. Survival curves were constructed via the Kaplan-Meier method and compared by log-rank test. Cox's proportional hazards model was used to identify independent factors associated with clinical outcomes. SPSS version 23.0 software (IBM Corp., Armonk, NY, USA) was used for analysis. $\mathrm{P}<0.05$ was considered to indicate a statistically significant difference.

\section{Results}

Patient characteristics. In our institution, 209 advanced RCC cases (134 males, 75 females) were treated with MTT between April 2008 and February 2017, i.e., after the Cytokine Era and before the initiation of 2nd generation immunotherapy. Some optional therapies were received in this cohort, such as radiotherapy ( $n=61$, mainly brain or bone), metasectomy $(n=19$, mainly lung), or previous immunotherapies ( $n=49$, interferon or interleukin-2). Median age was 67.2 [interquartile range (IQR): 60.0-72.9] years (Table I). Sorafenib, sunitinib, axitinib, pazopanib, temsirolimus, and everolimus were administered as 1st line MTT for 48, 54, 50, 47, 9, and 1 patient, respectively. In the cohort of all MTT lines (1st-7th lines), the median treatment days of sorafenib, sunitinib, axitinib, pazopanib, temsirolimus, and everolimus were $144.5(\mathrm{n}=74), 99.0(\mathrm{n}=79)$, $130.0(n=97), 60.5(n=74), 77.0(n=21)$, and 92 days $(n=76)$, respectively. Median RDI was $0.50,0.60,0.61,0.56,0.92$ and 0.56 , respectively. Nephrectomy was performed on 147 patients (70.3\%) before and after MTT. One, two, and more than three lines of MTTs were performed for 110 (52.6\%), 44 (21.1\%), and 55 patients (26.3\%), respectively.

\section{Impact of eTS on survival outcome in mRCC patients}

Conventional risk factors and survival outcome. Kaplan-Meier curves evaluating conventional IMDC risk factors for survival in mRCC patients of our cohort are shown in Fig. S1. In order to identify other prognostic predictive factors of OS, a Kaplan-Meier estimator curve of each factor was generated using the data of mRCC patients (Table I). Of note, all cohorts (Table I) included non-metastatic cases $(n=15)$ treated with MTT for locally advanced RCC (i.e., presurgical therapy). Sex (male vs. female, Fig. 1A), nephrectomized vs. not nephrectomized (Fig. 1B), IMDC risk classification (non-poor risk vs. poor risk, Fig. 1C), and four metastatic sites [the liver (Fig. 1D), bone (Fig. 1E), lymph nodes (Fig. 1F), and brain (Fig. 1G)] held proportional hazard assumptions, whereas age and three metastatic sites did not (data not shown). Among the factors with proportional hazards assumptions, Cox's proportional hazards model identified five independent predictors: IMDC risk classification (HR: 1.90), liver metastasis (HR: 2.08), bone metastasis (HR: 1.83), lymph node metastasis (HR: 1.75), and brain metastasis (HR: 3.19) ('mRCC patients' in Table II).

In sub-group analyses of the clear cell mRCC cohort (mccRCC, Table I), a Kaplan-Meier estimator curve of three risk factors [bone (Fig. 1H), lymph nodes (Fig. 1I), and brain (Fig. 1J)] held proportional hazards assumptions, whereas the others did not (data not shown). Cox's proportional hazards model revealed that all the factors with proportional hazards assumptions were independent predictors: Bone metastasis (HR: 2.08), lymph node metastasis (HR: 2.12), and brain metastasis (HR: 4.78) ('mccRCC patients' in Table II).

ETS and survival outcome. In the MTT era, as previously suggested $(11,16)$, eTS is another predictive factor in mRCC patients. Among mRCC patients, eTS data was available in 127 patients (Table I). Median days to initial radiographic 
Table I. Patient characteristics.

\begin{tabular}{|c|c|c|c|c|c|c|c|c|}
\hline \multirow[b]{2}{*}{ Variables } & \multicolumn{2}{|c|}{$\begin{array}{c}\text { Total } \\
\text { cohort }(n=209)\end{array}$} & \multicolumn{2}{|c|}{$\begin{array}{c}\mathrm{mRCC} \\
\text { patients }(\mathrm{n}=194)\end{array}$} & \multicolumn{2}{|c|}{$\begin{array}{c}\text { mccRCC } \\
\text { patients }(n=119)\end{array}$} & \multicolumn{2}{|c|}{$\begin{array}{c}\text { mRCC patients } \\
\text { with eTS data }(n=127)\end{array}$} \\
\hline & $\mathrm{n}$ & $\%$ & $\mathrm{n}$ & $\%$ & $\mathrm{n}$ & $\%$ & $\mathrm{n}$ & $\%$ \\
\hline \multicolumn{9}{|l|}{ Age } \\
\hline Median, IQR & 67.2 & $60.0-72.9$ & 67.1 & $60.0-72.8$ & 67.1 & $60.9-72.6$ & 66.9 & $59.2-72.8$ \\
\hline$<67$ & 106 & 50.7 & 99 & 51.0 & 61 & 51.3 & 69 & 54.3 \\
\hline$\geq 67$ & 103 & 49.3 & 95 & 49.0 & 58 & 48.7 & 58 & 45.7 \\
\hline \multicolumn{9}{|l|}{ Sex } \\
\hline Male & 134 & 64.1 & 125 & 64.4 & 81 & 68.1 & 85 & 66.9 \\
\hline Female & 75 & 35.9 & 69 & 35.6 & 38 & 31.9 & 42 & 33.1 \\
\hline Pathology & & & & & n.a. & & & \\
\hline Pure clear & 125 & 59.8 & 119 & 61.3 & & & 75 & 59.1 \\
\hline Non-pure clear & 32 & 15.3 & 26 & 13.4 & & & 15 & 11.8 \\
\hline Unknown & 52 & 24.9 & 49 & 25.3 & & & 37 & 29.1 \\
\hline IMDC risk classification & N.A. ${ }^{a}$ & & & & & & & \\
\hline Favorable & & & 7 & 3.6 & 4 & 3.4 & 5 & 3.9 \\
\hline Intermediate & & & 93 & 47.9 & 63 & 52.9 & 62 & 48.8 \\
\hline Poor & & & 75 & 38.7 & 38 & 31.9 & 51 & 40.2 \\
\hline Unknown & & & 19 & 9.8 & 14 & 11.8 & 9 & 7.1 \\
\hline \multicolumn{9}{|l|}{ Nephrectomy } \\
\hline Before MTT & 118 & 56.5 & 117 & 60.3 & 79 & 66.4 & 77 & 60.6 \\
\hline After MTT (presurgical) & 29 & 13.8 & 18 & 9.3 & 16 & 13.4 & 6 & 4.7 \\
\hline No & 62 & 29.7 & 59 & 30.4 & 24 & 20.2 & 44 & 34.6 \\
\hline \multicolumn{9}{|l|}{ Number of MTT lines } \\
\hline 1 & 110 & 52.6 & 98 & 50.5 & 58 & 48.7 & 58 & 45.7 \\
\hline 2 & 44 & 21.1 & 42 & 21.6 & 23 & 19.3 & 29 & 22.8 \\
\hline$\geq 3$ & 55 & 26.3 & 54 & 27.8 & 38 & 31.9 & 40 & 31.5 \\
\hline \multicolumn{9}{|l|}{ MTT in the 1 st line setting } \\
\hline Sorafenib & 48 & 23.0 & 47 & 24.2 & 25 & 21.0 & 30 & 23.6 \\
\hline Sunitinib & 54 & 25.8 & 54 & 27.8 & 33 & 27.7 & 34 & 26.8 \\
\hline Axitinib & 50 & 23.9 & 45 & 23.2 & 29 & 24.4 & 29 & 22.8 \\
\hline Pazopanib & 47 & 22.5 & 38 & 19.6 & 30 & 25.2 & 27 & 21.3 \\
\hline Temsirolimus & 9 & 4.3 & 9 & 4.6 & 2 & 1.7 & 6 & 4.7 \\
\hline Everolimus & 1 & 0.5 & 1 & 0.5 & 0 & 0.0 & 1 & 0.8 \\
\hline \multicolumn{9}{|l|}{ Metastatic sites } \\
\hline \multicolumn{9}{|l|}{ Number } \\
\hline 0 & 15 & 7.2 & & & & & & \\
\hline 1 & 73 & 34.9 & 73 & 37.6 & 52 & 43.7 & 43 & 33.9 \\
\hline 2 & 68 & 32.5 & 68 & 35.1 & 43 & 36.1 & 49 & 38.6 \\
\hline$\geq 3$ & 53 & 25.4 & 53 & 27.3 & 24 & 20.2 & 35 & 27.6 \\
\hline \multicolumn{9}{|l|}{ Location } \\
\hline Lung & 150 & 71.8 & 150 & 77.3 & 87 & 73.1 & 107 & 84.3 \\
\hline Liver & 25 & 12.0 & 25 & 12.9 & 10 & 8.4 & 16 & 12.6 \\
\hline Bone & 54 & 25.8 & 54 & 27.8 & 34 & 28.6 & 35 & 27.6 \\
\hline Lymph nodes & 84 & 40.2 & 84 & 43.3 & 47 & 39.5 & 54 & 42.5 \\
\hline Brain & 10 & 4.8 & 10 & 5.2 & 9 & 7.6 & 6 & 4.7 \\
\hline Pancreas & 12 & 5.7 & 12 & 6.2 & 5 & 4.2 & 8 & 6.3 \\
\hline Others & 51 & 24.4 & 51 & 26.3 & 26 & 21.8 & 33 & 26.0 \\
\hline \multicolumn{9}{|l|}{ Follow-up duration (months) } \\
\hline Median, IQR & 14.2 & $6.1-27.5$ & 14.1 & $6.0-27.6$ & 16.7 & $7.4-27.5$ & 16.7 & $6.3-27.5$ \\
\hline
\end{tabular}

Metastatic sites were counted on the day of the initiation of 1st line MTT. MTT, molecular targeted therapy; IQR, interquartile range; N.A., not available; IMDC, International Metastatic Renal Cell Carcinoma Database Consortium; mRCC, metastatic renal cell carcinoma; mccRCC, metastatic clear cell renal cell carcinoma; eTS, early tumor shrinkage; ${ }^{a}$ classified for only mRCC. 
A

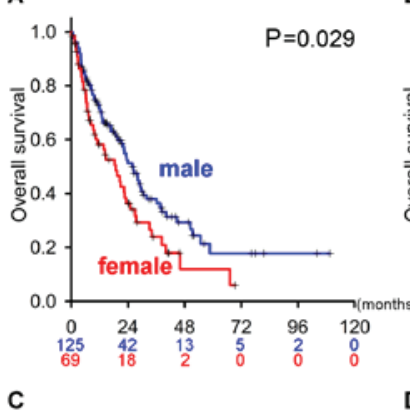

C

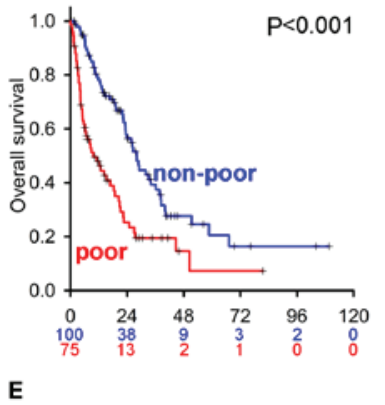

E

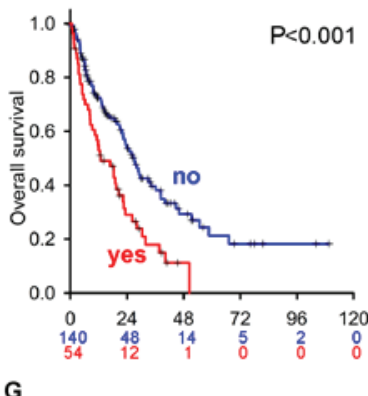

G

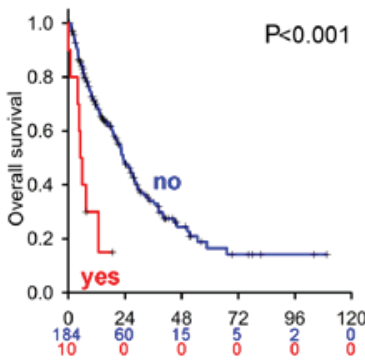

I

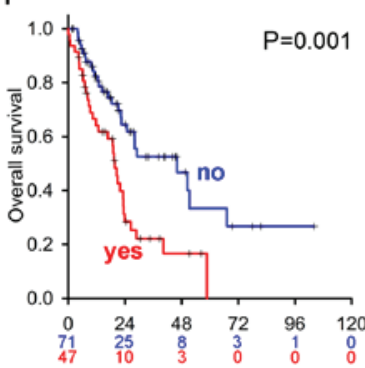

B

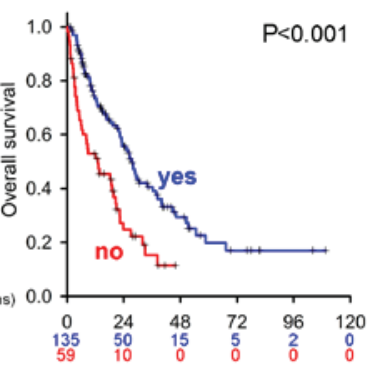

D
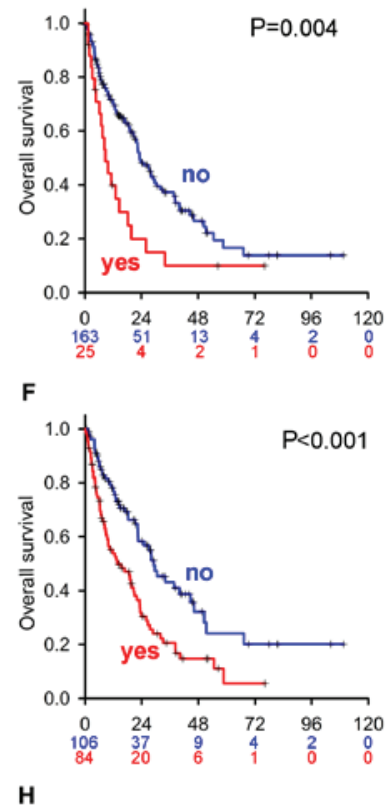

H

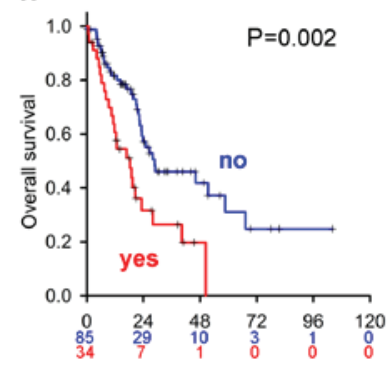

$\mathbf{J}$

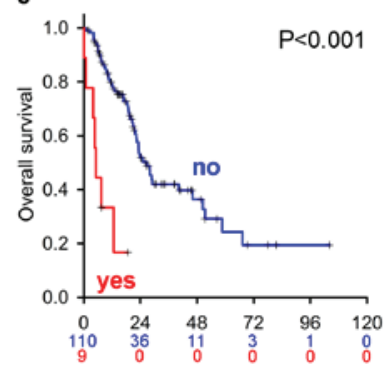

Figure 1. Kaplan-Meier survival curves of factors with proportional hazard assumptions in patients with mRCC and mccRCC. Kaplan-Meier curves of (A) sex (male vs. female), (B) nephrectomized (yes) vs. not nephrectomized (no), (C) non-poor risk vs. poor risk in the IMDC classification, (D) with or without liver metastasis (yes vs. no), (E) with or without bone metastasis (yes vs. no), (F) with or without lymph node metastasis (yes vs. no), and (G) with or without brain metastasis (yes vs. no) in all mRCC patient cohorts as well as $(\mathrm{H})$ with or without bone metastasis (yes vs. no), (I) with or without lymph node metastasis (yes vs. no), and ( $\mathrm{J}$ ) with or without brain metastasis (yes vs. no) in the mccRCC patient cohort. Blue and red numbers below the month in each graph indicate the number of patients in the groups represented by blue and red lines, respectively. IMDC, International Metastatic Renal Cell Carcinoma Database Consortium; mRCC, metastatic renal cell carcinoma; mccRCC, metastatic clear cell renal cell carcinoma.

evaluation after introduction of the drug were 60.0 days (IQR: 50.0-77.0). In these cases, measurable lesions were independently assessed by three physicians (HK, TO, and SM), the eTS value of each was calculated, and average eTS values were used in statistical analyses. The Friedman test revealed no significant differences in measurements between the three physicians $(\mathrm{P}=0.660)$, suggesting that these measurements were reliable. In order to estimate the predictive power of eTS, a Kaplan-Meier estimator curve of eTS with different cut-off values was generated (Fig. 2). Patients with eTS less than -0.2, $-0.15,-0.1,-0.05,0.0$, and +0.05 had better OS than those with larger cut-off values, suggesting that eTS is a potent predictor.

In the cohort of mRCC with eTS data (Table I), a Kaplan-Meier estimator curve of four risk factors [no preceding nephrectomy (Fig. 2G), liver metastasis (Fig. 2H), bone metastasis (Fig. 2I), and lymph node metastasis (Fig. 2J)] held proportional hazards assumptions, whereas age, sex, IMDC classification, brain metastasis, lung metastasis, pancreatic metastasis, and other metastases did not (data not shown). In Cox's proportional hazards models of these factors and eTS with different cut-off values, metastases of the liver and lymph nodes were independent predictive factors throughout the analyses (Table S1). As for eTS, cut-off values of $-0.15,-0.05$, and 0.0 were significant predictors. Overall, predictors estimating OS in the present study were proven to be consistent with those previously reported in a pre-MTT era cohort.

Difference in predictive factors between patients treated with different 1st line TKIs. In the cohort of mRCC patients with eTS data (Table I), sorafenib, sunitinib, axitinib, pazopanib, temsirolimus, and everolimus were administered as 1 st line MTT to 30 (23.6\%), 34 (26.8\%), 29 (22.8\%), 27 (21.3\%), $6(4.7 \%)$, and $1(0.8 \%)$ patient, respectively.

In order to visualize the power of predictors in each MTT, we delineated the Kaplan-Meier curves of all predictors in each 1st line MTT treatment cohort. However, due to the small number of cases in each MTT, no proportional hazards assumption was observed for any predictor, except eTS with two cut-off values $(0.0$ and +0.05$)$ in axitinib-treated patients (Fig. 3). Cox's proportional hazards model analyses revealed these HR values were very high at 3.52 (IQR: 1.32-9.36) and 4.39 (IQR: 1.56-12.37), respectively.

In sub-group analyses, axitinib was administered to 30 cases as 1 st line, 12 cases as 2 nd line, and 16 cases as 3 rd or more lines, respectively. Median OS of patients treated with 1st and 2 nd lines $(n=42)$ were similar to those treated with 3 rd or more lines (19.7 vs. 18.6 months, $\mathrm{P}=0.671$, respectively). Median progression free survival (PFS) of patients treated with the 1st and 2 nd lines $(n=42)$ was similar to that of patients treated with the 3 rd or more lines (12.1 vs. 11.4 months, $\mathrm{P}=0.620$, respectively). In patients treated with 1 st and 2 nd line axitinib therapy, eTS less than 0.0 resulted in significantly better OS $(\mathrm{P}=0.003)$ and PFS $(\mathrm{P}=0.006)$ than eTS greater than 0.0 .

\section{Discussion}

In the present study, we retrospectively identified prognostic predictive factors in several mRCC retrospective settings: mRCC patients including all pathological types $(n=194)$, mecRCC $(n=119)$, and mRCC with eTS data $(n=127)$. Four 


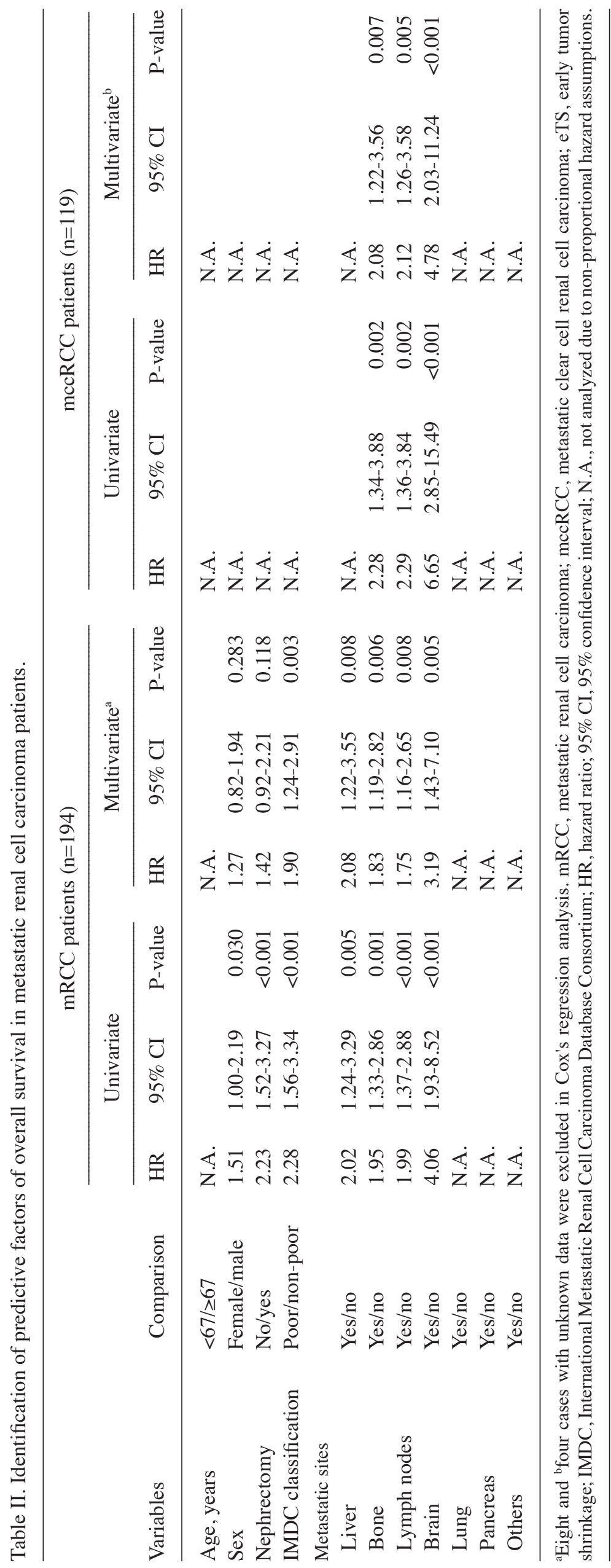



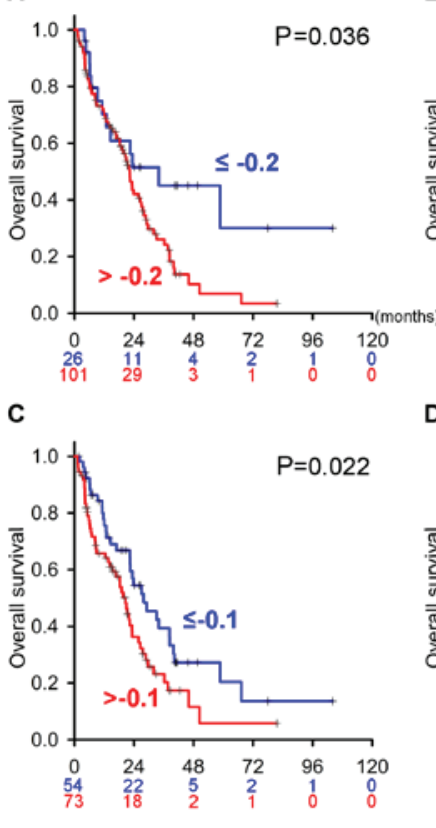

E

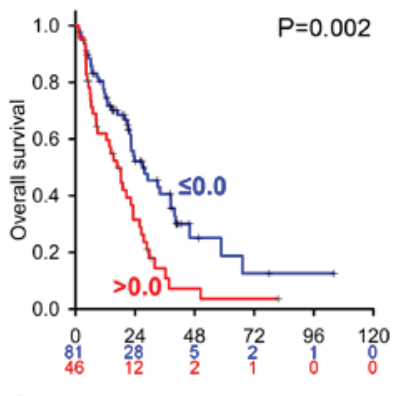

G
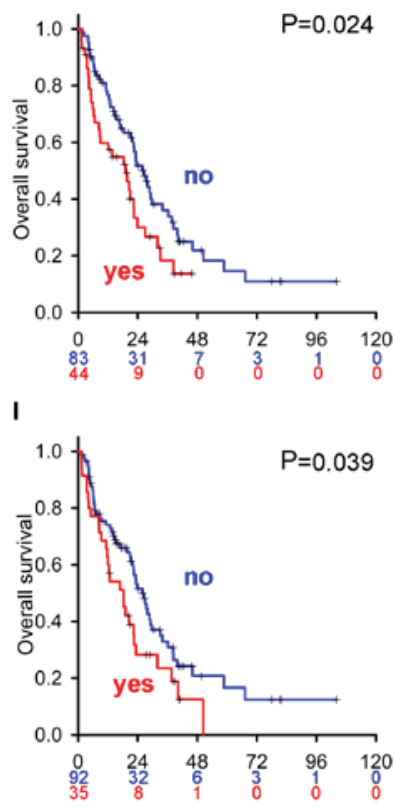

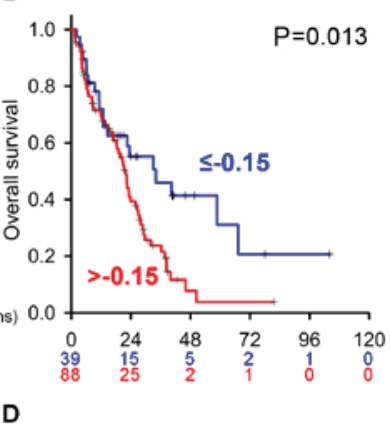

D
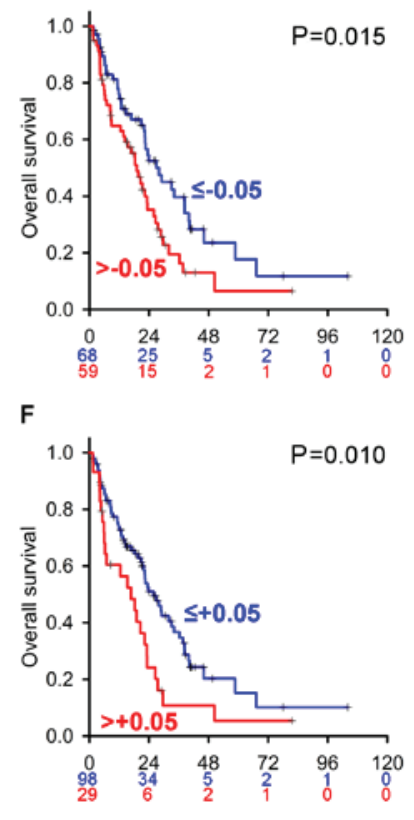

H

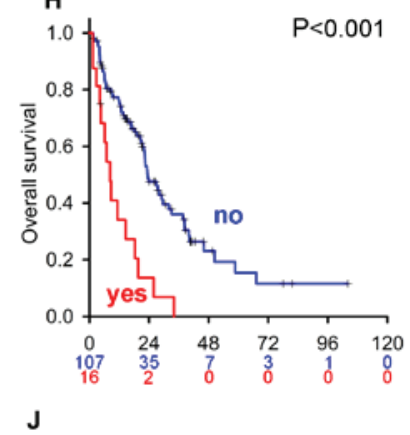

J

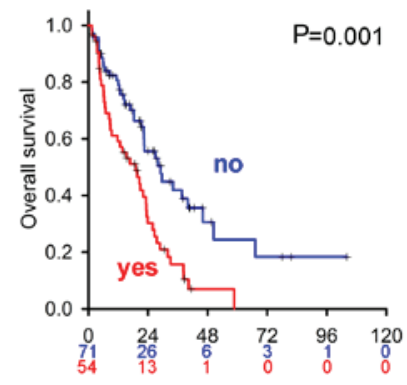

Figure 2. Kaplan-Meier survival curves of factors with proportional hazard assumptions in mRCC patients with eTS data. Kaplan-Meier curves of different eTS cut-off values: (A) $-0.2[\leq-0.2(>20 \%$ shrinkage) vs. $>-0.2(<20 \%$ shrinkage)], (B) -0.15 , (C) -0.1 , (D) -0.05 , (E) 0.0 and (F) +0.05 as well as (G) nephrectomized (yes) vs. not nephrectomized (no), (H) with or without liver metastasis (yes vs. no), (I) with or without bone metastasis (yes vs. no) and (J) with or without lymph node metastasis (yes vs. no) in the cohort of mRCC patients with eTS data $(n=127)$. Blue and red numbers below the month in each graph indicate the number of patients in the groups represented by blue and red lines, respectively. mRCC, metastatic renal cell carcinoma; eTS, early tumor shrinkage.
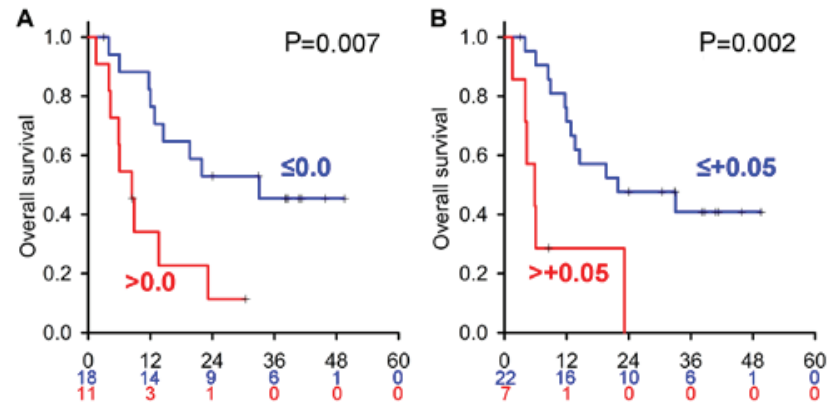

Figure 3. Kaplan-Meier survival curves of factors with proportional hazard assumptions in mRCC patients according to each MTT. Kaplan-Meier curves of different eTS cut-off values in axitinib (1st line)-treated patients: (A) eTS cut-off value: 0.0 , and (B) eTS cut-off value: -0.15 . Blue and red numbers below the month in each graph indicate the number of patients in the groups represented by blue and red lines, respectively. $\mathrm{mRCC}$, metastatic renal cell carcinoma; MTT, molecular targeted therapy; eTS, early tumor shrinkage.

metastatic sites in the liver, bone, lymph nodes, and brain as well as greater eTS were potentially independent predictors throughout these settings. Overall, these results were consistent with previous findings as described below.

Based on a Pubmed search, several studies were published on the usefulness of eTS. Rini et al (12) demonstrated that patients with eTS $\leq-0.1$ and administered axitinib were treatable for longer than 18 months and also had superior OS to those with eTS >-0.1. Similarly, Grünwald et al (13) reported that an eTS cut-off- 0.1 is a strong predictor of OS and PFS in a large scale $(\mathrm{n}=4,334)$ study. In a Japanese cohort, Miyake et al (11) found that eTS was an independent predictor of better OS in patients treated with 1st line sunitinib or sorafenib. Even in 2nd line treatments with axitinib, sunitinib, everolimus, temsirolimus, and sorafenib, eTS was a significant predictive factor of OS (13). In this study, treatment with axitinib resulted in earlier shrinkage than with other agents, thereby supporting the present results on axitinib. Overall, eTS appears to be a useful predictor of OS; however, further analyses are needed in order to select the best time to measure eTS (17).

The results of the present study suggest that eTS is advantageous for patients with axitinib. Axitinib is a selective inhibitor of VEGF receptors (18), and is currently used as 2nd line MTT (6). Since axitinib exhibits a potent shrinking ability, it is frequently administered as presurgical therapy (19). We used axitinib in many cases as 1st line MTT because we empirically hypothesized that axitinib shrunk mRCC faster than other TKIs (sorafenib, sunitinib, and pazopanib), possibly because of its high VEGF receptor affinity. Oya et al (20) recently reported that 1st line axitinib treatment is beneficial for Japanese mRCC patients, which supports our hypothesis. Our results showed that both OS and PFS of patients treated with 1st and 2nd lines were similar to those treated with 3 rd or more lines. We suggest that axitinib in any lines may reduce tumors in patients with mRCC, and that eTS is one of the predictors of OS in axitinib-treated patients.

There were some limitations in this study including its retrospective nature, limited number of patients in a single institution, and incomplete data collection. Our data set used in the current analysis included patients who received different lines of treatment and various agents, as well as those with or without some optional therapies such as cytoreductive nephrectomy, 
metasectomy, radiotherapy, and traditional immunotherapy, leading to heterogeneity in our cohort of patients.

We performed retrospective analyses to identify prognostic predictive factors of $\mathrm{mRCC}$ in the MTT era using a limited Japanese cohort in our single institution. Four metastatic sites in the liver, bone, lymph nodes, and brain appear to be independent predictors of OS throughout several cohorts, as previously reported. Furthermore, eTS was identified as a potent prognostic predictive factor, particularly with the administration of axitinib. Data from analyses using cases just before the immune checkpoint inhibitor era will add important evidence for MTT.

\section{Acknowledgements}

Not applicable.

\section{Funding}

The present study was supported in part by JSPS Grants-in-Aid for Scientific Research (grant no. JP17K18062).

\section{Availability of data and materials}

The datasets analyzed during the current study are available in the Figshare repository: All patients: doi. org/10.6084/m9.figshare.6972782.v1; eTS measurement: doi. org/10.6084/m9.figshare.6972788.v2.

\section{Authors' contributions}

SS and KN conceived and designed the study, and drafted the manuscript. HK, TO and SM retrospectively obtained the patient data. SS and RA analyzed the patient data. HK, TO, SM, TK, GK and MO revised the manuscript critically for important intellectual content. All authors read and approved the version to be published.

\section{Ethics approval and consent to participate}

The present study was approved by the Institutional Review Board of Saitama Medical University International Medical Center (approval no. 14-049); the requirement for written informed consent was waived due to the retrospective nature of this study.

\section{Patient consent for publication}

Not applicable.

\section{Competing interests}

The authors declare that they have no competing interests.

\section{References}

1. Hsieh JJ, Purdue MP, Signoretti S, Swanton C, Albiges L, Schmidinger M, Heng DY, Larkin J and Ficarra V: Renal cell carcinoma. Nat Rev Dis Primers 3: 17009, 2017.

2. Ratain MJ, Eisen T, Stadler WM, Flaherty KT, Kaye SB, Rosner GL, Gore M, Desai AA, Patnaik A, Xiong HQ, et al: Phase II placebo-controlled randomized discontinuation trial of sorafenib in patients with metastatic renal cell carcinoma. J Clin Oncol 24: 2505-2512, 2006.
3. Escudier B, Eisen T, Stadler WM, Szczylik C, Oudard S, Siebels M, Negrier S, Chevreau C, Solska E, Desai AA, et al: Sorafenib in advanced clear-cell renal-cell carcinoma. N Engl J Med 356: 125-134, 2007.

4. Naito S, Yamamoto N, Takayama T, Muramoto M, Shinohara N, Nishiyama K, Takahashi A, Maruyama R, Saika T, Hoshi S, et al: Prognosis of Japanese metastatic renal cell carcinoma patients in the cytokine era: A cooperative group report of 1463 patients. Eur Urol 57: 317-325, 2010.

5. Motzer RJ, Hutson TE, Tomczak P, Michaelson MD, Bukowski RM, Rixe O, Oudard S, Negrier S, Szczylik C, Kim ST, et al: Sunitinib versus interferon alfa in metastatic renal-cell carcinoma. N Engl J Med 356: 115-124, 2007.

6. Rini BI, Escudier B, Tomczak P, Kaprin A, Szczylik C, Hutson TE, Michaelson MD, Gorbunova VA, Gore ME, Rusakov IG, et al: Comparative effectiveness of axitinib versus sorafenib in advanced renal cell carcinoma (AXIS): A randomised phase 3 trial. Lancet 378: 1931-1939, 2011.

7. Motzer RJ, Hutson TE, Cella D, Reeves J, Hawkins R, Guo J, Nathan P, Staehler M, de Souza P, Merchan JR, et al: Pazopanib versus sunitinib in metastatic renal-cell carcinoma. $\mathrm{N}$ Engl J Med 369: 722-731, 2013.

8. Motzer RJ, Escudier B, Oudard S, Hutson TE, Porta C, Bracarda S, Grünwald V, Thompson JA, Figlin RA, Hollaender N, et al: Efficacy of everolimus in advanced renal cell carcinoma: A double-blind, randomised, placebo-controlled phase III trial. Lancet 372: 449-456, 2008.

9. Hudes G, Carducci M, Tomczak P, Dutcher J, Figlin R, Kapoor A, Staroslawska E, Sosman J, McDermott D, Bodrogi I, et al: Temsirolimus, interferon alfa, or both for advanced renal-cell carcinoma. N Engl J Med 356: 2271-2281, 2007.

10. Motzer RJ, Escudier B, McDermott DF, George S, Hammers HJ, Srinivas S, Tykodi SS, Sosman JA, Procopio G, Plimack ER, et al: Nivolumab versus everolimus in advanced renal-cell carcinoma. N Engl J Med 373: 1803-1813, 2015.

11. Miyake H, Miyazaki A, Imai S, Harada K and Fujisawa M: Early tumor shrinkage under treatment with first-line tyrosine kinase inhibitors as a predictor of overall survival in patients with metastatic renal cell carcinoma: A retrospective multi-institutional study in Japan. Target Oncol 11: 175-182, 2016.

12. Rini BI, Gruenwald V, Jonasch E, Fishman MN, Tomita Y, Michaelson MD, Tarazi J, Cisar L, Hariharan S, Bair AH, et al: Long-term duration of first-line axitinib treatment in advanced renal cell carcinoma. Target Oncol 12: 333-340, 2017.

13. Grünwald V, Lin X, Kalanovic D and Simantov R: Early tumour shrinkage: A tool for the detection of early clinical activity in metastatic renal cell carcinoma. Eur Urol 70: 1006-1015, 2016.

14. Heng DY, Xie W, Regan MM, Warren MA, Golshayan AR, Sahi C, Eigl BJ, Ruether JD, Cheng T, North S, et al: Prognostic factors for overall survival in patients with metastatic renal cell carcinoma treated with vascular endothelial growth factor-targeted agents: Results from a large, multicenter study. J Clin Oncol 27: 5794-5799, 2009.

15. Eisenhauer EA, Therasse P, Bogaerts J, Schwartz LH, Sargent D, Ford R, Dancey J, Arbuck S, Gwyther S, Mooney M, et al: New response evaluation criteria in solid tumours: Revised RECIST guideline (version 1.1). Eur J Cancer 45: 228-247, 2009.

16. Miyake H, Harada K, Ozono S and Fujisawa M: Prognostic significance of early tumor shrinkage under second-line targeted therapy for metastatic renal cell carcinoma: A retrospective multi-institutional study in Japan. Mol Diagn Ther 20: 385-392, 2016.

17. Ishihara H, Yagisawa T, Kondo T, Omae K, Takagi T, Iizuka J, Kobayashi $\mathrm{H}$ and Tanabe $\mathrm{K}$ : Effect of the timing of best tumor shrinkage on survival of patients with metastatic renal cell carcinoma who received first-line tyrosine kinase inhibitor therapy. Int J Clin Oncol 22: 126-135, 2017.

18. Rixe O, Bukowski RM, Michaelson MD, Wilding G, Hudes GR, Bolte O, Motzer RJ, Bycott P, Liau KF, Freddo J, et al: Axitinib treatment in patients with cytokine-refractory metastatic renal-cell cancer: A phase II study. Lancet Oncol 8: 975-984, 2007.

19. Karam JA, Devine CE, Urbauer DL, Lozano M, Maity T, Ahrar K, Tamboli P, Tannir NM and Wood CG: Phase 2 trial of neoadjuvant axitinib in patients with locally advanced nonmetastatic clear cell renal cell carcinoma. Eur Urol 66: 874-880, 2014.

20. Oya M, Tomita Y, Fukasawa S, Shinohara N, Habuchi T, Rini BI, Fujii Y, Kamei Y, Umeyama Y, Bair AH and Uemura H: Overall survival of first-line axitinib in metastatic renal cell carcinoma: Japanese subgroup analysis from phase II study. Cancer Sci 108: 1231-1239, 2017. 